\title{
Optimization of Electrical Networks Modes on Transformation Coefficients of Transformers
}

\author{
Tulkin Gayibov ${ }^{*}$, Sherkhon Latipov ${ }^{2}$, and Davron Abdurashidov ${ }^{3}$ \\ 1,2Tashkent State Technical University named after Islam Karimov, Uzbekistan \\ ${ }^{3}$ Department of Power engineering, Karshi Engineering-Economics Institute, \\ Kashkadarya, Uzbekistan
}

\begin{abstract}
Optimization of modes of electrical networks provides for the determination of rational values of reactive powers of controlled sources, voltages of reference nodes and transformation coefficients of adjustable loop transformers, at which the minimum costs associated with the production, transmission and distribution of electricity are ensured and all the specified operating and technological constraints are met. As a result of a lot of work performed by specialists from all over the world on the development of methods and algorithms for optimization the modes of electrical networks, at present, the methods for optimization of reactive powers and voltages of nodes are quite developed. At the same time, the algorithms for optimization of transformation coefficients of transformers, taking into account the provision of permissible voltage levels and minimum energy losses in closed electric networks by ensuring the optimal distribution of power flows in them, requires corresponding improvement. In this regard, this work proposes a new algorithm for optimization of modes of electrical networks on transformation coefficients of adjustable loop transformers. The results of researched the effectiveness of the proposed algorithm are presented.
\end{abstract}

\section{Introduction}

The goal of the optimization problem for electrical networks modes is to determine the optimal values of all adjustable parameters - reactive powers or voltages of nodes with reactive power sources, transformation coefficients of adjustable transformers in the range of permissible modes. The area of permissible operating modes of electric network is determined by conditions of balance of powers in nodes of electrical network, constraints in the form of inequalities on boundary permissible values of active power of the balancing node, voltages of all nodes, reactive power of controlled sources and transformation coefficients of adjustable transformers. To date, as the results of the work of many scientists and specialists around the world, many methods and algorithms for solving the problem under consideration have been developed. They effectively use various approaches and optimization methods, such as classical $[1,2,13$, $14]$, heuristic [3, 4], artificial intelligence [5, 6, 7], etc.

Most of the above mentioned works provide for the optimization of modes of electrical networks on reactive power or voltage of power plants and reactive power compensators [5-7, 12]. At the same time, it is possible to separate a number of works, in particular, [8-11], which have made a significant contribution to the development of the theory of optimization on transformation coefficients of transformers.

According to the algorithm described in [8], the choice of optimal transformation coefficient of transformer with corresponding control taps is based on optimization of voltage in fictitious node on the secondary side. Evaluation of effectiveness of using this interesting algorithm for optimization of modes of complex-closed electrical networks, from our point of view, may encounter difficulties in taking into account functional constraints in the form of inequalities in the calculation process. In [9], an algorithm for choosing the optimal control taps of transformers based on the by particle swarm optimization method is given. However, according to the materials presented in it, the procedure for taking into account various simple and functional constraints in the form of inequalities in the optimization process remains unclear. The work [10] describes an algorithm for optimization the mode of an electrical network based on minimization of losses in closed networks by opening the circuits and compensating for reactive power. The algorithm given in [11] provides for the selection of optimal transformation coefficients of transformers according to the criterion of ensuring admissible voltage levels at consumers. Therefore, this algorithm cannot be directly used to minimize losses in electrical networks.

Transformers in closed electrical networks can be used not only to regulate the voltage in them, but also to minimize losses by changing the distribution of power flows in parallel networks.

Thus, the issues of improving of algorithms for optimization the modes of closed electrical networks on transformation coefficients of adjustable transformers, taking into account all types of operating and technological constraints, remain as a important problem. This paper proposes the algorithm for optimization of modes of electrical networks on transformation coefficients of adjustable loop transformers, which reduce losses in electrical networks to a minimum by ensuring optimal power flows at the area of permissible modes.

\footnotetext{
* Corresponding author: tulgayibov@gmail.com
} 


\section{Optimization method}

The minimum losses in closed electrical networks can be ensured through the optimal distribution of power flows in them. This can be achieved by creating additional electromotive forces (EMF) in independent loops of closed networks. The creation of such EMF can be achieved on the basis of compensation of reactance or by adjusting the transformation coefficients of adjustable transformers [12]. Usually, the second activity is the most effective. Determination of the optimal transformation coefficients of such transformers for complex-closed networks, taking into account all types of constraints emanating from the conditions for ensuring admissible modes, in particular admissible voltage levels, is a complex problem of nonlinear mathematical programming.

In special cases, this problem can be solved on the basis of a two-fold calculation of steady state of the electrical network. In this case, one proceeds from the condition of optimality of the natural distribution of power flows in closed networks, the elements of which have only active resistances [12]. At first time the calculation of steady state of electrical network is carried out taking into account only the active resistances of its elements (Fig. 1, a). As a result, the power flows in all branches, including the branch with a regulated transformer, of electrical network are determined. Then at the point of location of adjustable transformer the circuit is opened. In this case, in the nodes obtained as a result of opening, the power flows along this branch, obtained as a result of calculating the steady state, taking into account only active resistances, are deposited. After that, the second time calculation of steady state of electrical network, taking into account the impedances of all elements and with an open loop is carried out (Fig. 1, b). The ratio of obtained voltages of nodes, which appeared as a result of the circuit opening, at this stage gives the optimal transformation coefficient of the adjustable transformer, at which the minimum losses in the closed electrical network are provided (Fig. 1, v).

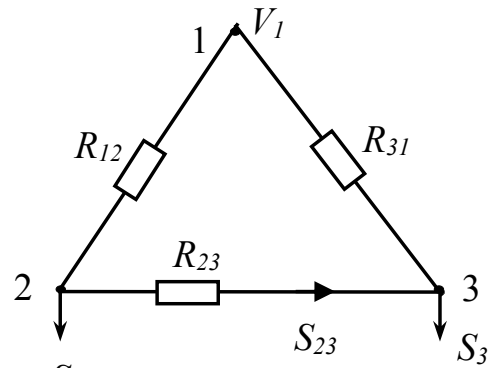

$S_{2}$ a)
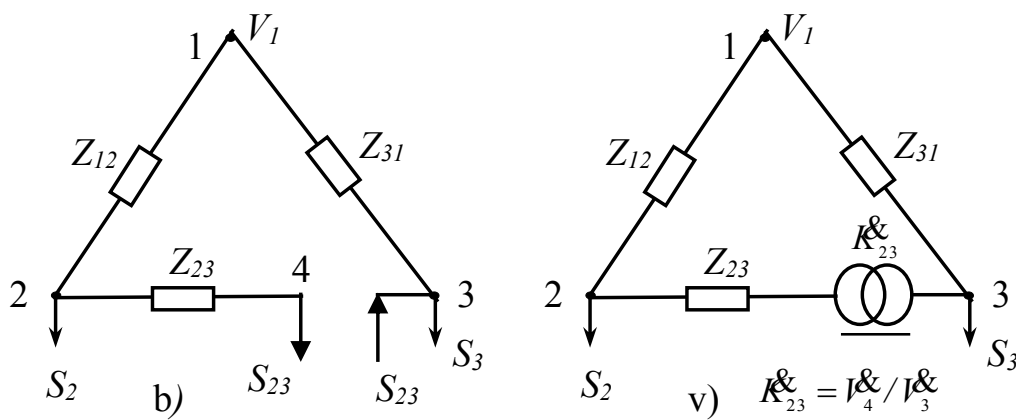

Fig. 1. Determination of optimal transformation ratio of the loop transformer for a simple closed electrical network.

In general cases, the above-described algorithm for optimization of transformation coefficients of transformers cannot be applied to complex-closed electrical networks with many different constraints in the form of inequalities. In such cases, the algorithm described below can be effectively used.

The objective function is a function of total losses of active power in electrical networks, defined as a algebraic sum of the active powers of all nodes. And the active power of the node, in turn, is described as the sum of the active powers of power flows along the branches outgoing from it:

$$
\pi=\sum_{i=0}^{n} P_{i}=\sum_{i=0}^{n} \sum_{j \in J_{i}} P_{i j} \rightarrow \text { min }
$$

The task is to determine the optimal transformation coefficients of adjustable loop transformers, at which the minimum of function (1) is provided, taking into account the constraints on the balance of active and reactive power of nodes

$$
\begin{aligned}
& W_{i}^{\prime}=P_{i}-\bar{P}_{i}=0, \quad i \in G+N ; \\
& W_{i}^{\prime}=Q_{i}-\bar{Q}_{i}=0, \quad i \in G+N-G_{u} ;
\end{aligned}
$$

minimum and maximum limit values of the active power of the balancing power plant

$$
P_{0}^{\min } \leq P_{0} \leq P_{0}^{\max }
$$

reactive power of reference nodes

$$
Q_{i}^{\min } \leq Q_{i} \leq Q_{i}^{\max }, \quad i \in G_{u} \text {; }
$$

voltages of all nodes

$$
V_{i}^{\min } \leq V_{i} \leq V_{i}^{\max }, \quad i \in G+N
$$

individual components of the complex transformation coefficients of adjustable loop transformers (in the general case)

$$
\left.\begin{array}{l}
K_{l}^{\prime \min } \leq K_{l}^{\prime} \leq K_{l}^{\prime \text { max }} \\
K_{l}^{\prime \prime \text { min }} \leq K_{l}^{\prime \prime} \leq K_{l}^{\prime \prime m a x}
\end{array}\right\}, \quad l \in T_{k} \text { : }
$$

power flows and currents of power transmission lines (PTL) with observed power flow and current

$$
\begin{array}{ll}
P_{l}^{\text {min }} \leq P_{l} \leq P_{l}^{\text {max }}, & l \in L_{P} ; \\
I_{l}^{\text {min }} \leq I_{l} \leq I_{l}^{\text {max }}, & l \in L_{I},
\end{array}
$$

where $\mathrm{n}$ is the number of nodes in the electrical network (except for the balancing one); $J_{i}$ is a set of nodes that have direct connections with node $i ; N, G$ are sets of nodes with loadings and generating units; $G_{u}$ is a set of reference (reactive power generating) nodes; $T_{K}$ is a set of branches containing transformers with adjustable transformation coefficients; $L_{P}, L_{I}$ are the set of PTL in which the active power flows and currents are observed; $P_{i}, \bar{P}_{i}, Q_{i}, \bar{Q}_{i}$ are determined and given active and reactive power of the $i$ th node; $K^{\prime}, K^{\prime \prime}{ }_{l}$ are real and imaginary components of transformation coefficient of a transformer with longitudinal-transverse regulation; $P_{l}, I_{l}$ are determined flow of active power and current of the $l$ th power line.

The active and reactive powers of nodes are expressed through modules and phase angles of the complex voltages of nodes $V_{i}, V_{j}, \delta_{i}, \delta_{j}$; real and imaginary components of intrinsic and mutual conductivities of nodes $i$ and $j-g_{i i}, b_{i i}, g_{i j}, b_{i j}$, as well as taking into account the designation $\delta_{i j}=\delta_{i}-\delta_{j}$ as

$$
\begin{aligned}
& P_{i}=\sum_{j \in J_{i}} P_{i j}=g_{i i} V_{i}^{2}-V_{i} \sum_{j \in J_{i}} V_{j}\left(g_{i j} \cos \delta_{i j}-b_{i j} \sin \delta_{i j}\right), \\
& Q_{i}=\sum_{j \in J_{i}} P_{i j}=-b_{i i} V_{i}^{2}-V_{i} \sum_{j \in J_{i}} V_{j}\left(g_{i j} \sin \delta_{i j}+b_{i j} \cos \delta_{i j}\right),
\end{aligned}
$$


The $p-q$ branch in the circuit of an electrical network containing a adjustable transformer is represented in the form shown in Fig. 2 .

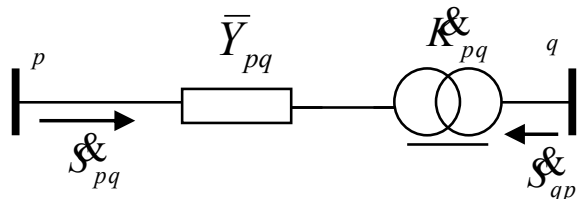

Fig. 2. Representation of a branch with an adjustable transformer.

In Fig. $2 \bar{Y}_{p q}=g_{p q}+j b_{p q}$ is the total complex conductance of the branch, which has the corresponding active and reactive components and include the conductance of the transformer; $K_{p q}^{\&}=K_{p q}^{\prime}+j K_{p q}^{\prime \prime}$ is the complex transformation coefficient of the ideal transformer with the corresponding components.

The power fluxes along the branch with an adjustable transformer $\oiint_{p q}^{\&}, \oiint_{q p}^{\&}$ (Fig. 1) and their components $P_{p q}, P_{q p}$ are expressed through the conductivity of the branch $\bar{Y}_{p q}$, modules and phase angles of complex voltages of nodes $V_{p}, V_{q}, \delta_{p}, \delta_{q}$ and the optimized transformation coefficient of transformer. At each step of iterative optimization process, there is a change in transformation coefficient of transformer by $\Delta K_{p q}=\Delta K_{p q}^{\prime}+j \Delta K_{p q}^{\prime \prime}$ and a corresponding change in the power flows by $\Delta P_{p q}, \Delta Q_{p q}, \Delta P_{q p}, \Delta Q_{q p}$, which are expressed through $\Delta K_{p q}^{\prime}, \Delta K_{p q}^{\prime \prime}$ and $V_{p}, V_{q}, \delta_{p}, \delta_{q}$.

As a result of changes of power flows in the branch the total losses of active power in networks also change:

$$
\pi=\sum_{i=0}^{n} P_{i}+\Delta P_{p q}+\Delta P_{q p}
$$

The solution of the obtained problem of conditional minimization of function (11), taking into account the restrictions described above, according to the proposed algorithm, for see reducing it to the problem of unconditional minimization of the following generalized function

$$
F=\pi+P F+\sum_{i \in G+N} \mu_{i}^{\prime} W_{i}^{\prime}+\sum_{i \in G+N-G_{u}} \mu_{i}^{\prime \prime} W_{i}^{\prime \prime},
$$

where

$P F=P F_{0}+\sum_{i \in G_{u}} P F_{Q_{i}}+\sum_{i \in G+N} P F_{U i}+\sum_{l \in L p} P F_{P_{l}}+\sum_{l \in L_{l}} P F_{I_{l}}$ is the sum of penalty functions taking into account constraints (3), (4)., (5), (7) and (8), respectively; $\mu_{i}^{\prime}, \mu_{i}^{\prime \prime}$ are indefinite Lagrange multipliers.

At each iteration a step in the direction of decreasing the generalized function (12) by gradient method

$$
\begin{aligned}
& K_{p q}^{\prime(k)}=K_{p q}^{\prime(k-1)}-h_{p q}^{\prime(k)} \cdot{\frac{\partial F^{(k)}}{\partial K_{p q}^{\prime}}}^{(k)} \\
& K_{p q}^{\prime \prime(k)}=K_{p q}^{\prime \prime(k-1)}-h_{p q}^{\prime(k)} \cdot{\frac{\partial F^{(k)}}{\partial K_{p q}^{\prime \prime}}}^{{ }^{\prime \prime}}
\end{aligned}
$$

and calculation of steady state of electrical network by the NewtonRaphson method at obtained transformation coefficient of transformer are made.

\section{Results and discussions}

The effectiveness of the algorithm proposed here for several examples, in particular, in example of optimization of mode of electrical network, the circuit of which is shown in Fig. 3, on transformation coefficients of adjustable transformers in branches 1-2 and 5-4 was researched. The active $r$ and inductive $x$ resistances of the branches are given in Ohms $(\Omega)$, and the total complex nodal powers are given in MVA. Node 0 is balancing node, the voltage of which is $220 \mathrm{kV}$. The ranges of control taps on the high sides of the transformers are the same and have $\pm 6 \times 2 \%$. In series to the transformers in branches 1-2 and 5-4 the regulated transformers, which could also rotate the voltage phases on the secondary side, are connected. The range of possible values of real and imaginary components of transformer coefficients of transformers connected in series in branches 1-2 and 5-4 are the same and $1.5 \leq \mathrm{K}^{\prime} \leq 2.5$ and $-0.5 \leq \mathrm{K}^{\prime} \leq .0 .5$.

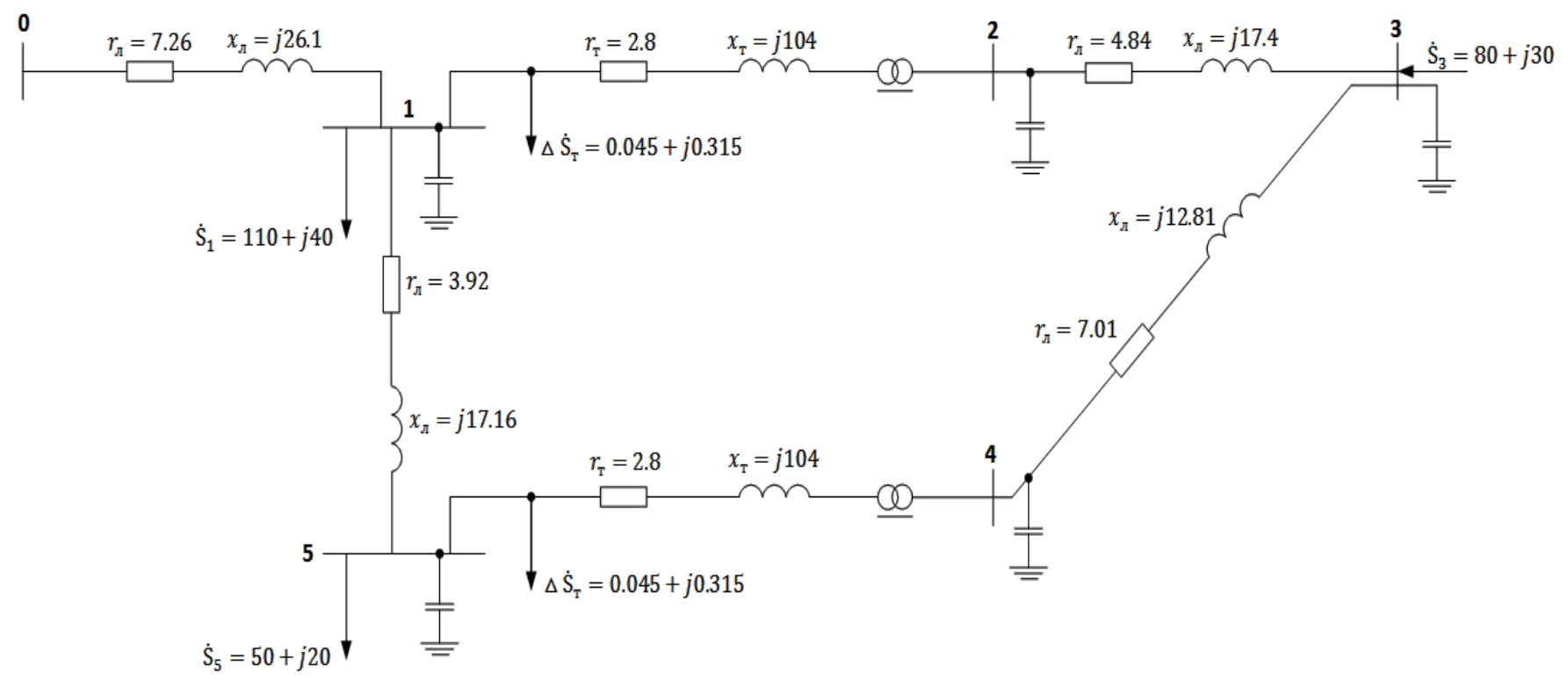

Fig. 3. The circuit of the electrical network.

To evaluate the optimization results by the proposed algorithm based on comparison, at first the initial steady-state mode of the electrical network was calculated for the initial values of transformation coefficients of transformers $K_{12}=K_{54}=1$.9. The 
components of the complex voltages of nodes obtained as a result of such a calculation are shown in Table 1. The total losses of active power in electrical network are 3.60 MW.

Table 1. Parameters of the initial steady state mode of electrical network.

\begin{tabular}{|c|c|c|}
\hline Node number, $i$ & $V_{i}, \mathrm{kV}$ & $\delta_{i}, \mathrm{rad}$. \\
\hline 0 & 220.00 & 0.0 \\
\hline 1 & 215.65 & -0.0261 \\
\hline 2 & 118.59 & 0.0825 \\
\hline 3 & 124.11 & 0.1354 \\
\hline 4 & 118.30 & 0.0968 \\
\hline 5 & 215.53 & -0.0236 \\
\hline
\end{tabular}

The results of optimization of electrical network mode on complex transformation coefficient of loop transformer in branch 1-2 are shown in Table 2. In this case, the optimal transformation coefficient is $K_{12.0 p t .}=1.838+\mathrm{j} 0.139\left(K_{12}^{\prime}=1.838\right.$ and $K^{\prime \prime}{ }_{12}=$ 0.139 ). The total losses of active power in electrical network decreased to $3.45 \mathrm{MW}$. Thus, the reduction of losses due to optimization is $0.15 \mathrm{MW}$, i.e. $4.2 \%$.

Table 2. Parameters of the optimal mode of electrical network on transformation coefficient of transformer in branch 1-2.

\begin{tabular}{|c|c|c|}
\hline Node number, $i$ & $V_{i}, \mathrm{kV}$ & $\delta_{i}, \mathrm{rad}$. \\
\hline 0 & 220.00 & 0.0 \\
\hline 1 & 215.59 & -0.0260 \\
\hline 2 & 120.54 & 0.0309 \\
\hline 3 & 125.65 & 0.0958 \\
\hline 4 & 119.98 & 0.0693 \\
\hline 5 & 215.87 & -0.0278 \\
\hline
\end{tabular}

In order to assess the effect of transformers in branches 1-2 and 5-4 on the total losses in electrical networks, optimizations were also made on transformation coefficient of transformer in branch 5 4 and simultaneously on the transformation coefficients of transformers in both branches 1-2 and 5-4. Table 3 shows the obtained optimal transformation coefficients of transformers and the corresponding total losses of active power in electrical networks.

Table 3. Total losses of active power in electrical networks at optimal transformation coefficients of transformers in branches 1-2 and 5-4.

\begin{tabular}{|c|c|c|c|c|}
\hline $\begin{array}{c}\text { Optimized } \\
\text { transformation } \\
\text { coefficients }\end{array}$ & $K_{l-2}$ & $K_{5-4}$ & $\begin{array}{c}\text { Total } \\
\text { losses } \\
\pi, \mathrm{MW}\end{array}$ & $\begin{array}{c}\text { Reduction } \\
\text { of losses, } \\
\%\end{array}$ \\
\hline Initial mode & 1.9 & 1.9 & 3.60 & - \\
\hline$K_{I 2}$ & $\begin{array}{c}1.838+ \\
+\mathrm{j} 0.139\end{array}$ & 1.9 & 3.45 & 4.2 \\
\hline$K_{54}$ & 1.9 & $\begin{array}{c}1.769- \\
-\mathrm{j} 0.098\end{array}$ & 3.34 & 7.0 \\
\hline$K_{I 2}, K_{54}$ & $\begin{array}{c}1.5+ \\
+\mathrm{j} 0.046\end{array}$ & $\begin{array}{c}1.5- \\
-\mathrm{j} 0.037\end{array}$ & 2.44 & 32.1 \\
\hline
\end{tabular}

Table 4. Distribution of power flow in branch of electrical network according to transformer coefficients of loop transformer.

\begin{tabular}{|c|c|c|c|}
\hline Modes & $\begin{array}{c}\text { Optimized } \\
\text { transformer } \\
\text { coefficients }\end{array}$ & $\begin{array}{c}\text { Power flow } \\
S_{2 I}=P_{2 I}+j Q_{2 l}, \\
\text { MVA }\end{array}$ & $\begin{array}{c}\text { Reduction } \\
\text { of losses, } \\
\%\end{array}$ \\
\hline Initial & - & $50.81+\mathrm{j} 16.59$ & - \\
\hline Optimal on $K_{I 2}$ & $K_{12}$ & $60.95+\mathrm{j} 8.85$ & 4.2 \\
\hline Optimal on $K_{54}$ & $K_{54}$ & $61.25+\mathrm{j} 23.20$ & 7.0 \\
\hline $\begin{array}{c}\text { Optimal on } K_{12} \\
\text { and } K_{54}\end{array}$ & $K_{12}$ and $K_{54}$ & $61.20+\mathrm{j} 15.68$ & 32.1 \\
\hline
\end{tabular}

On the basis of calculation experiments was set that power losses reduction in closed networks occurs due to changing of power distribution in them. To approve this factor in table 4 power flow in branch (in direction from node 2 to 1 ) in initial and optimized on transformer coefficients modes are presented.

As a result of carried out researches also revealed that the phase rotation of the complex voltage of the node under the influence of complex transformation coefficient of the loop transformer more strongly affects the redistribution of power flows in closed networks and thereby expands the possibilities of significantly reducing the losses in them. The degree of influence of the transformation coefficient of a loop transformer on the redistribution of power flows in closed networks and, accordingly, on power losses in them, significantly depends on the location of the transformer in the network. The increase in the number of regulated transformers in closed networks expands the possibilities for obtaining an additional effect by reducing losses in them.

\section{Conclusion}

1. A new effective algorithm for optimization of modes of electrical networks on transformation coefficients of adjustable loop transformers is proposed. It allows to reliably determine the optimal complex transformation coefficients of loop transformers taking into account all types of operating and technological limitations.

2. A decrease in the total losses of active power in closed electrical networks when optimizing their modes on transformation coefficients of loop transformers occurs due to the optimal redistribution of power flows under the influence of the created loop EMF.

3. Rotation of the voltage phase under the influence of the complex transformation coefficient is a determining factor for the redistribution of power flows in closed networks and for corresponding reduction of losses in electrical networks. In addition, the degree of influence of transformation coefficient to total losses in electrical networks significantly depends on the location of the regulated transformer. Therefore, it is recommended to determine the rational places for installation of adjustable transformers in electrical networks taking into account this factor.

\section{References}

1. Zhu, J.: Optimization of Power System Operation. Wiley-IEEE Press (2015).

2. Yang, X.S.: Nature-inspired metaheuristic algorithms. pp. 11108, Luniver Press (2010).

3. Michalewicz, Z., Fogel, D.B.: How to Solve It. Modern Heuristics. pp. 145-487, Springer (2004).

4. Colorni, A., Dorigo, M., Maniezzo, V.: Distributed Optimization by Ant Colonies. In: Proceedings of ECAL91, pp. 134-142. Elsevier Publishing, Paris (1991), pp. 134-142, 1991.

5. A. Kartikeya Sarma, K. Mahammad Rafi. (2011). Optimal Selection of Capacitors for Radial Distribution Systems Using Plant Growth Simulation Algorithm. International Journal of Advanced Science and Technology. 30 (5), 43-54.

6. M.H. Sulaiman, Z. Mustaffa, M.R. Mohamed, O. Aliman, Using the gray wolf optimizer for solving optimal reactive power dispatch problem, Appl. Soft Comput. J. 32 (2015) 286-292,

http://dx.doi.org/10.1016/j.asoc.2015.03.041.

7. S. Mouassa, T. Bouktir, Artificial bee colony algorithm for discrete optimal reactive power dispatch, in: Proc. 2015 Int. Conf. Ind. Eng. Syst. Manag, IEEE IESM 2015, 2016. http://dx.doi.org/10.1109/IESM.2015.7380228.

8. B. A. Robbins, H. Zhu and A. D. Domínguez-García, "Optimal Tap Setting of Voltage Regulation Transformers in Unbalanced Distribution Systems," in IEEE Transactions on Power Systems, 
vol. 31, no. 1, pp. 256-267, Jan. 2016, doi: 10.1109 / TPWRS.2015.2392693.

9. Vadim Manusov, Pavel Matrenin, Nasrullo Khasanzoda. Power Loss Minimization by Voltage Transformer Turns Ratio Selection based on Particle Swarm Optimization. Przeglad Elektrotechniczny. August, 2019. doi: 10.15199 / 48.2019.08.28.

10. L. Ramesh, S. P. Showdhury, S. Showdhury, A. A. Natarajan, C. T. Gaunt. Minimization of Power Loss in Distribution Networks by Different Techniques. International Journal of Electrical, Computer, Energetic, Electronic and Communication Engineering. Vol: 3, No: 4, 2009.

11. Dauren Akhmetbayev, Arman Akhmetbayev, Aigerim Aidarova. Determination of rational transformation coefficients of transformers distribution networks. E3S Web of Conferences 25, 04003 (2017). RSES 2017. DOI: 10.1051 / e3sconf / 20172504003.
12. Nasyrov T.Kh., Gayibov T.Sh. Theoretical foundations of optimization of power systems modes. - T .: "Fan va technology", 2014. (in Russian).

13. T. Gayibov, "The algorithm of Power Systems Short period regime optimization through short-linear approximation of nonlinear dependences," Proceedings of the 2011 3rd International Youth Conference on Energetics (IYCE), Leiria, 2011, pp. 1-3.

14. Tulkin Gayibov, Sherkhon Latipov, Bakhadir Uzakov. Power System Mode optimization by piecewise-linear approximation of energy characteristics of Power Plants. In: Rudenko International Conference "Methodological problems in reliability study of large energy systems" E3S Web of Conference, 139, 01086. RSES, Irkutsk, Russia (2019).

doi: https://doi.org/10.1051/e3sconf/201913901086 